\title{
Research \\ Possible risk factors for the formation of antisperm antibodies in a subfertile population
}

\author{
Varuni Tennakoon ${ }^{1}$, Surangi G Yasawardene ${ }^{2}$, Deepal S Weerasekera ${ }^{1}$ \\ Sri Lanka Journal of Obstetrics and Gynaecology 2011; 33: 12-19
}

\begin{abstract}
with pelvic inflammatory disease (PID) (20\%)

\section{Objectives:}

1. To investigate the incidence of antisperm antibodies (ASA) in a subfertile population in a Sri Lankan set up.

2. To determine the possible risk factors for the formation of ASA among them.

than PID free females $(8 \%)$.

Conclusion: Genital surgeries of men play a significant role in formation of ASA in males. Females who have undergone IUIs and who have had episodes of PID are at a greater risk in developing ASA.
\end{abstract}

Methods: A prospective descriptive study was carried out on subfertile couples treated at 'Prarthana', Centre for ART, during the period from 01.01.2006 to 01.01.2009. A total of 230 couples were included. Using an investigator administered questionnaire following information was obtained for each subject demographic details, medical, surgical histories, genito-urinary infections, illnesses and medications, social habits etc. Presence of ASA was elicited using mixed antiglobulin reaction latex bead test (SpermMAR, Fertipro $\mathrm{NV}$, Belgium) on spermatozoa, seminal plasma and serum of males and cervical mucus, serum and follicular fluid of females.

Results: Out of 230 couples, 48 (20.86\%) were positive for ASA. There were no significant associations observed between the presence of ASA and age, duration of marriage/subfertility, occupation, type of subfertility, associated autoimmune diseases, illnesses, and use of addictables in both males and females. Of the seven males who had undergone genital surgeries two were positive for ASA (Chi$\mathrm{sq}=4.407$, P-value=0.036). A proportionately higher incidence of ASA was observed in females $(11.7 \%)$ who had previous intra-uterine insemination (IUI) compared to females who did not have IUIs (5.88\%). Markedly higher association of ASA was observed in females

${ }^{1}$ Prarthana Centre for ART, Rajagiriya, Colombo. ${ }^{2}$ Department of Anatomy, Faculty of Medical Sciences, University of Sri Jayewardenepura, Nugegoda.

Correspondence: Varuni Tennakoon

E-mail:varuni16email@yahoo.com
Key words: antisperm antibodies, incidence, risk factors.

\section{Introduction}

Antisperm antibodies (ASA) are naturally occurring antibodies against sperm antigens. It is established that ASA can negatively impact fertility by affecting sperm motility, cervical mucus penetration, gamete fusion and potentially even the first steps of embryo development ${ }^{1}$. ASA can be found in systemically (in blood and lymph) and in local secretions (in semen, cervical-vaginal, uterine, oviductal and follicular fluid) ${ }^{2}$. The precise mechanism of generation of these antibodies is yet to be discovered. When spermatozoa are produced at puberty they express antigens not previously encountered by immune system. These immunogenic spermatozoa, in spite of the protective mechanisms to avoid an immune response, may induce autoimmune and isoimmune responses in male and female. Theoretically, breach of the immune-regulatory mechanisms in human reproductive system may lead to exposure of immunogenic sperm antigens to immune system resulting in an inflammatory reaction and formation of $\mathrm{ASA}^{3}$. However, the formation of ASA does not occur in every individual who has a breach of immune-regulatory mechanisms. This paper discusses the associated risk factors for the formation of ASA in subfertile males and females.

\section{Objectives}

The objectives of the present study were,

1. To investigate the incidence of ASA in a subfertile population in a Sri Lankan set up.

2. To determine the possible risk factors for the formation of ASA among them. 
The present study also compares the findings with those already published in literature and enhances our understanding of ASA that may well lead to the identification and treatment implication of such couples.

\section{Materials and methods}

This prospective descriptive study commenced in January 2006 and subfertile couples, who presented for treatment at the 'Prarthana', Centre for ART, 1175, Cotta Road, Rajagiriya were recruited up to January 2009. The total study population was 460 individuals with 230 males and 230 females. The subfertile couples who volunteered to participate were interviewed by the investigator after obtaining informed written consent. The ethical clearance for the study was obtained from the Ethical Review Committee of the Faculty of Medical Sciences, University of Sri Jayawardenepura, Gangodawila, Nugegoda, Sri Lanka.

The investigator administered questionnaire was filled for both males and females. Age of the individual, duration of marriage, type of subfertility and duration of subfertility were noted. The medical and surgical histories were obtained. More concern was paid to obstetrics and gynaecological histories and procedures in the female. History of mumps, genitourinary infections and surgeries/procedures, trauma to genitalia were obtained from the male. The current illnesses or any medication that the individual was on was also asked. A special emphasis was paid to illnesses and diseases of autoimmune origin including hypothyroidism, hyperthyroidism, rheumatoid arthritis, psoriasis etc. Details of the occupational and social history were also taken at the interview. Social habits such as consumption of alcohol and smoking were questioned. In relevant subjects, the quantity and frequency of substance usage were noted.

Presence of ASA was tested both in males and females. In the male spermatozoa, seminal plasma and serum were checked for ASA isotypes of IgA and IgG. In the female cervical mucus and serum were checked for both $\operatorname{IgA}$ and $\operatorname{IgG}$ isotypes of ASA and in addition, females who underwent IVF treatment at Prarthana, the oocyte free follicular fluid was also checked for both types of ASA.

The ASA were detected using mixed antiglobulin reaction latex bead test (SpermMAR, Fertipro NV, Belgium). In mixed antiglobulin reaction (MAR) assay/ the 'SpermMAR' test, which is a commercially available test, have IgA and IgG coated latex particles to detect ASA. The particles are mixed with washed viable sperms and agglutination in the presence of ASA is observed by light microscopy. This test provides a rapid assay time, good specificity, isotype and the location of the ASA and the ability to use viable sperms. The direct 'SpermMAR' test was performed on spermatozoa and indirect test was performed on seminal plasma, cervical mucus, serum and follicular fluid to elicit ASA. The percentage of motile sperm exhibiting latex bead binding was calculated. A test with $>30 \%$ of the sperms with bound beads was considered positive for $\mathrm{ASA}^{14}$.

Chi square test was used to analyze statistical significance of the presence of ASA with demographic details and other information of subfertile males and females.

\section{Results}

\section{Incidence of antisperm antibodies}

The incidence of antisperm antibodies among the subfertile couples of the study sample was $20.87 \%$ $(n=48,48 / 230)$. Antisperm antibody incidence among the males in the sample was $12.61 \%(n=29,29 / 230)$ while for the females it was $8.26 \%(n=19,19 / 230)$.

\section{Age}

In the total study population, the age range for the males was 27 to 49 years and for the females the age range was 21 to 40 years. The mean age \pm SD of the males was $36.9 \pm 5.0$ years and the mean age \pm SD for the females was $33.4 \pm 4.1$ years.

Males and females were grouped in 5 year age groups as shown in Table 1. There was no significant association observed between the presence of ASA and age factor in both males (Chi-sq $=3.532, \mathrm{P}$-value $=0.473$ ) and females (Chi-sq=0.039, P-value=0.998).

Table 1. Age distribution and pattern of ASA distribution in relation to the age of females and males in total study population

\begin{tabular}{|c|c|c|c|c|c|c|c|}
\hline Age groups (years) & & $20-24$ & $25-29$ & $30-34$ & $35-39$ & $40-44$ & $>45$ \\
\hline Total no. of females & 04 & $(01.7 \%)$ & $37(16.1 \%)$ & $96(41.7 \%)$ & $72(31.3 \%)$ & $21(9.1 \%)$ & 0 \\
\hline Total no. of ASA $(+)$ females & & 0 & $03(8.1 \%)$ & $08(8.3 \%)$ & $06(8.3 \%)$ & $02(9.5 \%)$ & 0 \\
\hline Total no. of males & & 0 & $13(5.7 \%)$ & $60(26.1 \%)$ & $93(40.4 \%)$ & $41(17.8 \%)$ & $23(10 \%)$ \\
\hline Total no. of ASA $(+)$ males & & 0 & $01(7.7 \%)$ & $06(10 \%)$ & $14(15.1 \%)$ & $06(14.6 \%)$ & $02(8.7 \%)$ \\
\hline
\end{tabular}




\section{Duration of marriage and duration of subfertility}

The duration of marriage ranged from six months to more than ten years. There were 11 couples who were married for six months to one year and 15 couples who were married for more than ten years at the time of recruiting for the study. The mean duration of marriage was 5.8 years. There was no significant association between the presence of ASA and duration of marriage (Chi-sq=8.45, P-value=0.207). Interestingly, there were no ASA positives among the couples who were married for six months to one year period. The distribution of ASA with the duration of marriage is shown in Table 2.

The duration of subfertility ranged from six months to 19 years. There were 27 couples having subfertility for less than one year and 15 couples having subfertility for more than ten years. The mean duration of subfertility was 5.3 years. There was no significant association found between the presence of ASA and duration of subfertility (Chi-sq=7.750, Pvalue $=0.257)$. The distribution of ASA with the duration of subfertility is shown in Table 2.

\section{Occupation}

The occupation of males and females were grouped into seven categories for the statistical analysis. The percentage of males exposed to heat, vibration or radiation was $4.78(n=11)$. It was observed that none of these subjects were positive for ASA. The incidence of ASA in different occupational categories was analyzed and no significant association noted in both males (chi-sq=6.064, P-value=0.300) and females. The pattern of distribution of ASA in different occupational categories is shown for males and females in Table 3.

Table 2. The distribution of ASA with the duration of marriage and duration of subfertility

\begin{tabular}{ccccc}
\hline Duration (years) & \multicolumn{2}{c}{ Marriage } & \multicolumn{2}{c}{ Subfertility } \\
\hline & Total no. of couples & $\begin{array}{c}\text { Total no. of ASA } \\
(+) \text { couples }\end{array}$ & Total no. of couples & $\begin{array}{c}\text { Total no. of ASA } \\
(+) \text { couples }\end{array}$ \\
\hline$<1$ & $11(4.8 \%)$ & 0 & $27(11.7 \%)$ & $04(14.8 \%)$ \\
$>1-2$ & $22(9.6 \%)$ & $03(13.6 \%)$ & $33(14.4 \%)$ & $06(18.2 \%)$ \\
$>2-4$ & $58(25.2 \%)$ & $10(17.2 \%)$ & $57(24.8 \%)$ & $08(14.1 \%)$ \\
$>4-6$ & $60(26.1 \%)$ & $17(28.3 \%)$ & $55(23.9 \%)$ & $18(32.7 \%)$ \\
$>6-8$ & $37(16.1 \%)$ & $11(29.7 \%)$ & $27(11.7 \%)$ & $06(22.2 \%)$ \\
$>8-10$ & $27(11.7 \%)$ & $05(18.5 \%)$ & $16(7.0 \%)$ & $04(25 \%)$ \\
$>10$ & $15(6.5 \%)$ & $02(13.3 \%)$ & $15(6.5 \%)$ & $02(13.3 \%)$ \\
\hline
\end{tabular}

Table 3. Occupational categories and pattern of distribution of ASA among categories in males and females

\begin{tabular}{lllll}
\hline Occupational category & $\begin{array}{c}\text { Total no. } \\
\text { of males }\end{array}$ & $\begin{array}{c}\text { Total no. of ASA } \\
(+) \text { males }\end{array}$ & $\begin{array}{c}\text { Total no. of } \\
\text { females }\end{array}$ & $\begin{array}{c}\text { Total no. of ASA } \\
(+) \text { females }\end{array}$ \\
\hline Professionals & $18(7.8 \%)$ & $01(5.6 \%)$ & $18(7.8 \%)$ & $01(5.6 \%)$ \\
Managers, clerical & $115(50 \%)$ & $14(12.2 \%)$ & $84(36.5 \%)$ & $08(9.5 \%)$ \\
Skilled workers & $12(5.2 \%)$ & 0 & $01(0.4 \%)$ & 0 \\
Service personnel & $07(3.0 \%)$ & $01(14.3 \%)$ & $01(0.4 \%)$ & 0 \\
Businessmen & $64(27.8 \%)$ & $12(18.8 \%)$ & $11(4.8 \%)$ & 0 \\
Other & $14(6.1 \%)$ & $01(7.1 \%)$ & $04(1.7 \%)$ & 0 \\
Non employed & 0 & 0 & $111(48.3 \%)$ & $10(9.1 \%)$ \\
\hline
\end{tabular}




\section{Type of subfertility}

Of the study population, $76 \%(n=174)$ couples had primary subfertility while $24 \%(n=56)$ couples had secondary subfertility. The incidence of ASA with the type of subfertility was analyzed and showed no significant association between them (Chi-sq=0.765, $\mathrm{P}$-value $=0.382$ ). The incidence of ASA among primary and secondary subfertile couples was $19.54 \%(n=34)$ and $25 \%(n=14)$ respectively.

\section{Associated genito-urinary conditions in males}

The conditions and surgeries that would breach the blood testis barrier and facilitate formation of ASA were taken into consideration. Of the study sample $3.04 \%(n=7)$ males had undergone genital surgeries such as varicocoelectomy and inguinal herniorrhaphy. Out of these males two $(28.57 \%)$ were positive for ASA compared to the $12.12 \%$ ASA positivity among subjects $(n=27)$ without history of genital surgery. It was observed as a statistically significant (Chi-sq $=4.407$, $\mathrm{P}$-value $=0.036$ ) association between the presence of ASA and a history of genital surgery. Testicular trauma, orchidectomy, vasectomy or spinal cord injuries were not observed in males in the study sample.

\section{Associated autoimmune conditions and illnesses in males}

It was observed that $27.83 \%(n=64)$ of the males had mumps in childhood although none had mumps orchitis. Of the males who had mumps during childhood, $17.19 \%(n=11)$ were positive for ASA. Incidence of ASA with childhood mumps was analyzed and no significant association (Chi- $\mathrm{sq}=1.687, \mathrm{P}$-value=0.194) was found. None of the males gave a history of rheumatoid arthritis, autoimmune thyroid disease or any other systemic autoimmune disease.

Other co-morbid illnesses as hypertension, hypercholesterolemia, diabetes mellitus - type II etc. were observed in $10.43 \%(n=24)$ of males. Incidence of ASA with these illnesses was analyzed, and no significant association was found. The related data are shown in Table 4.

\section{Use of addictables in males}

Of the total sample $67.8 \%(n=156)$ of the males have never or very occasionally smoked or consumed alcohol. Although $31.3 \%(n=72)$ of the males consumed alcohol, no one admitted that they were addicted. It was observed that $13 \%(n=30)$ of the males were smokers. There was no one who was addicted to other addictables such as heroin. The incidence of ASA in the groups consuming addictables and not consuming addictables was analyzed and no significant association (Chi-sq=0.982, P-value=0.322) was found. Data is shown in Table 4.

\section{Associated factors in females}

From the study sample, $40.87 \%(n=94)$ of females had experienced intra-uterine insemination (IUI) with prepared sperms before recruitment for the study. Out of them $11.7 \%(n=11)$ were positive for ASA. Though there was no statistically significant relationship found, the incidence of ASA was proportionately higher among women those who had previous IUIs $(11.7 \%)$ compared to the women who did not have IUIs (5.88\%).

Table 4. Incidence of ASA with childhood mumps, co-morbid illnesses and use of addictables (alcohol and cigarette)

\begin{tabular}{lcc}
\hline & Total no. of males & Total no. of ASA (+) males \\
\hline History of childhood mumps & $64(27.8 \%)$ & $11(17.2 \%)$ \\
No history of childhood mumps & $166(72.2 \%)$ & $10(18.8 \%)$ \\
With co-morbid illnesses & $24(10.4 \%)$ & $04(16.7 \%)$ \\
Without co-morbid illnesses & $206(89.6 \%)$ & $25(12.2 \%)$ \\
& $74(32.2 \%)$ & $07(09.5 \%)$ \\
Use of addictables & $156(67.8 \%)$ & $22(14.1 \%)$ \\
No use of addictables & & \\
\hline
\end{tabular}


Table 5. Incidence of ASA with previous intra uterine insemination (IUI), pelvic inflammatory disease (PID), termination of pregnancy, autoimmune diseases and co-morbid illnesses in females

\begin{tabular}{lcc}
\hline & Total no. of females & Total no. of ASA (+) females \\
\hline History of previous IUI & $94(40.8 \%)$ & $11(11.7 \%)$ \\
No history of previous IUI & $136(59.1 \%)$ & $08(05.9 \%)$ \\
History of PID & $05(02.2 \%)$ & $01(20 \%)$ \\
No history of PID & $225(97.8 \%)$ & $18(08 \%)$ \\
History of termination of pregnancy & $56(24.4 \%)$ & $04(07.1 \%)$ \\
No history of termination of pregnancy & $176(76.5 \%)$ & $15(08.6 \%)$ \\
With autoimmune diseases & $21(09.1 \%)$ & 0 \\
Without autoimmune diseases & $209(90.9 \%)$ & $19(09.1 \%)$ \\
With co-morbid illnesses & $09(03.9 \%)$ & $02(22.2 \%)$ \\
Without co-morbid illnesses & $221(96.1 \%)$ & $17(07.7 \%)$ \\
\hline
\end{tabular}

There were 05 females who admitted having had episodes of pelvic inflammatory disease (PID) and proved by the documentation done at each treatment cycles. Out of these five females, one was positive for ASA. Therefore the incidence of ASA among females having PID was $20 \%$ compared to incidence in PID free females $8 \%(18 / 225)$. Markedly higher association of ASA was observed in females with PID.

It was observed that $24.3 \%(n=56)$ of females had a history of termination of pregnancy (spontaneous or induced) and among them $7.14 \%(n=04)$ were positive for ASA. There was no significant association found between the presence of ASA and history of termination of pregnancy.

It was also observed that $9.1 \%(n=21)$ had autoimmune diseases as autoimmune thyroid diseases, asthma, rheumatoid arthritis and psoriasis. However, non of these females were positive for ASA. Another $3.9 \%(n=9)$ had co-morbid illnesses as hypertension, hypercholesterolemia, diabetes mellitus - type II and hyperprolactenemia. Out of these females $22.22 \%(n=02)$ were positive for ASA. There was no significant association between presences of ASA and co-morbid illnesses.

None of the females of the study sample were smokers or consumed alcohol or any other addictables. The related data is shown in Table 5.

\section{Discussion}

Sperm have foreign antigens because they are not present until after puberty. According to Burnet's clonal selection theory, the cells bearing foreign antigens ultimately are destroyed by antibodies, when exposed to the immune system of the human body ${ }^{5}$. Since, breach of immune protective mechanisms do not cause formation of ASA in every individual and all individuals with ASA do not posses those interferences, to understand the etiology of ASA formation, exploration of other possible risk factors is needed. Epidemiological studies on ASA related immune-subfertility in Sri Lanka have been performed only infrequently. The present study shows an incidence of $20.87 \%$ ASA among subfertile couples with $12.61 \%$ in males and $8.26 \%$ in females. This corresponds with world data, which has been estimated to range from $9-36 \%$ in subfertile couples depending on the test format, detecting assays and the reporting centre ${ }^{1}$. A study done in Sri Lanka reported the incidence of ASA among the total sample was $9.5 \%$; among males it was $8.25 \%$ while that of females was $1.75 \%$ when detected by SpermMAR test in semen of males and serum of both males and females ${ }^{6}$. Compared to that study, the higher incidences could be explained that more test samples were performed on each subject to screen ASA in the present study. The present study showed a higher incidence of ASA in males than the females. Although the males had a proportionately higher incidence, it 
was not statistically significant with the incidence of females. Many studies have shown similar results ${ }^{6,7}$.

A trend towards late marriages at present contributes for higher mean age of males and females in subfertile population than in the past. The mean age of the females was 33.38 years and that of males was 36.86 years in the present study. It corresponds with most of published literature ${ }^{8}$. Another reason for the higher mean age of the study sample would be due to the late presentation of subfertile couples for the treatment in assisted reproductive technologies. The present study did not show a significant difference in incidence among the age groups that were analyzed in both males and females. It can be explained by the fact that the whole study population fell into the reproductive age group and was a selective population of subfertile couples. The presented results therefore demonstrated that there is no age dependent tendency in incidence of ASA during the reproductive age among subfertile couples. Different studies have shown the existence of age dependence in the incidence of natural sperm antibodies in normal animals $^{9}$ and that such variation exists in human as well ${ }^{10}$. However, these studies have employed predominantly healthy individuals and demonstrated conflicting results. Another study has shown a low incidence of ASA in prepubertal ages, a rise during reproductive age with a gradual decline with aging ${ }^{11}$.

There was no statistically significant difference observed between the incidence of ASA and duration of marriage in the present study. The same result was observed with duration of subfertility as well. Interestingly, there were no ASA positives observed among the couples $(n=11)$ who were married for six months to one year period. Mechanical or chemical disruption of the mucosal layer of the female genital tract may permit exposure to foreign sperm antigens and, ultimately ASA formation ${ }^{12}$. This may explain the absence of ASA in females in early part of marriage. However, the reason most women do not develop an immune response after repeated sperm exposure is not yet established. The other possible explanation for this observation would be the small number of couples that were investigated in that particular duration of marriage would have demonstrated that result by chance. The lack of local or foreign data in relation to the incidence of ASA with duration of marriage or subfertility makes it impossible to compare the data of the present study.

Occupation of the male partner has been widely studied in relation to the state of subfertility. However, the impact on immune-subfertility by the type of occupation is not clear. Occupational exposure to lead or mercury was found to make proteins better antigens. Shamy et al. have assessed serum ASA levels in healthy male workers exposed to lead or mercury and demonstrated ASA in $90 \%$ of workers exposed to lead
$84.6 \%$ of workers exposed to mercury ${ }^{13}$. No subjects were exposed to heavy metals in the present study and the incidence of ASA in different occupational categories was not significant. Factors such as heat, vibration, radiation and stress have been suggested as risk factors for subfertility although its impact on immune-subfertility is questionable.

Presence of ASA can be found in primary as well as secondary subfertility in both males and females. In the present study, there was no significant difference found in the incidence of ASA between primary subfertility and secondary subfertility. ASA may prevail in both types of subfertility in both males and females as it could develop with time, influenced by other conditions.

It was observed as a statistically significant association $(\mathrm{P}$-value $=0.036)$ between the presence of ASA and a history of genital surgery in the present study. Majority of them had undergone varicocoelectomy. Theoretically, presence of varicocoeles can also induce formation of ASA as impaired venous drainage of the testis may result in damage to the seminiferous tubules and lead to ASA production and the surgery may alleviate that, though causing disruption of blood testis barrier. Hence, it is highly recommended to screen subfertile males with a history of varicocoelectomy/any genital surgery for ASA in initial fertility workup. It is shown that after vasectomy, approximately $50 \%$ of men produce ASA $^{14}$. Likewise, a disproportionately higher incidence of ASA has been shown in testicular injured men ${ }^{15}$, spinal cord injured men ${ }^{16}$ and men with varicocoeles/varicocoelectomies ${ }^{17}$.

Inflammation may lead potentially to genital tract disruption and ASA formation. History of mumps has been shown to cause abnormal semen parameters in a Sri Lankan population ${ }^{18}$. However, formation of ASA is basically caused by mumps orchitis. Absence of a history of genital tract infections was a significant observation in the present study. Anyhow, no semen samples were found to be positive for culture in men who had increased pus cells and microorganisms. In the present study it was observed that $27.83 \%$ of the subfertile males had mumps in childhood, and none had mumps orchitis. Incidence of ASA with childhood mumps was not significant.

In recent studies some authors have suggested a possible cross reactivity of antibodies to spermatozoa in individuals who are having a systemic autoimmune disease. A study by Shirashi et al. has shown the incidence of ASA in males with systemic autoimmune diseases $(7.1 \%)$ was significantly higher than in the healthy controls $(0 \%)$. They have concluded that systemic autoimmune diseases may be one of the risk factors for developing ASA in men ${ }^{19}$. However, in the present study, neither the males gave a history of 
rheumatoid arthritis, autoimmune thyroid disease or any other systemic autoimmune disease, nor the females gave a history of any systemic autoimmune illness were positive for ASA. Hence, a statistical analysis could not be made with the presence of ASA with autoimmune illnesses. This observation may have occurred due to small number of subjects with autoimmune diseases in the study sample. Further studies are needed in this regard.

In the present study there was no statistically significant association found with the presence of comorbid illnesses in males and females. A definite conclusion of the effect of a co-morbid illness or its drugs in formation of ASA cannot be made since the number of subjects with the illness was small. Effect of some antihypertensives, anticonvulsants and antipsychotics are known to cause impairment of fertility by affecting the spermatogenesis or by sexual dysfunction $^{20}$. However, the co-morbid illnesses like hypertension, epilepsy, hyperprolactinaemia and diabetes mellitus type II have not been studied to determine the association with the incidence of ASA in large scale studies.

In the present study there was no significant correlation found with smokers and non-smokers in the incidence of ASA. By reviewing the literature on impact of smoking, alcohol and other addictables on immune-subfertility, it is evident that the effects remain inconclusive. Studies on cigarette smoking and incidence of ASA are limited. A study by Ludwikowski et al. has shown an increased number of ASA in smoking patients, though statistically not significant ${ }^{21}$. The data on alcohol show an apparent protective effect of moderate alcohol drinking on sperm parameters, probably due to the antioxidant effect of some alcoholic beverages ${ }^{22}$. In one study, users of cigarettes, marijuana or alcohol showed no difference in prevalence of ASA compared to non-users ${ }^{23}$. The present study too shares the same results; there was no significant correlation found with alcohol consumption and incidence of ASA.

In females another possible contributory factor for the formation of ASA is repeated intrauterine inseminations (IUI) with washed spermatozoa. IUI is widely used for the treatment of subfertility as a result of cervical or male factors or empirically before in vitro fertilization. In the present study, though there was no statistically significant relationship found, the incidence of ASA was proportionately higher among women who had previous IUIs $(11.7 \%)$ compared to the women who did not have IUIs $(5.88 \%)$. There is a theoretical concern that IUI may induce ASA in women since in IUI, semen bypasses the physiological immune barrier of female and washing removes immune-suppressive properties of seminal plasma. However, different studies have shown that IUI does not induce significant ASA production in women ${ }^{24}$.
In the present study, out of five females who have had episodes of pelvic inflammatory disease (PID), only one was positive for ASA. The incidence of ASA among females having PID was $20 \%$ while that of in PID free females was $8 \%$. Though the association of ASA with PID is markedly high, sufficient number of subjects is needed to make a statistical analysis. Krause and Naz have stated that infections of the genital tract as a cause of formation of ASA, has not sufficiently supported by clinical studies so far $^{25}$. However, some authors have demonstrated a direct association of PID with the incidence of ASA in women ${ }^{26}$.

Considering all the associated factors that have been investigated, it is evident that genital surgeries of men play a significant role in formation of ASA. Likewise, women who have undergone IUIs and who have had episodes of PID are at a greater risk in developing ASA. It is strongly recommended to screen such individuals for the presence of ASA in initial subfertility workup to direct them to appropriate treatment methodologies saving time, money and emotional stress of those subfertile couples. Also, larger scale studies are in need to establish the association with autoimmune diseases, co-morbid illnesses and drugs on formation of ASA.

\section{Acknowledgements}

This study was carried out with the financial support from the National Science Foundation, Sri Lanka. Research Grant No. RG/2007/HS/02.

\section{References}

1. Lee R, Goldstein M, Ullery BW, Witkin SS, et al. Value of serum antisperm antibodies in diagnosing obstructive azoospermia. Journal of Urology 2009; 181: 264-69.

2. Francavilla F, Santucci R, Barbonetti A, Francavilla S. Naturally occurring antisperm antibodies in men: interference with fertility and clinical implications. An update. Frontiers in Bioscience 2007; 12: 290-1.

3. Diekman AB, Norton EJ, Westbrook VA, et al. Antisperm antibodies from infertile patients and their cognate sperm antigens: a review. Identify between SAGA-1, the H6-3C4 antigen and CD 52. Am J Reprod Immunol 2000; 43: 134-43.

4. World Health Organization. WHO laboratory manual for the examination of human semen and sperm cervical mucus interaction 1999, Fourth edition, Cambridge University Press.

5. Burnet FM. The cllonal selection theory of acquired immunity. Cambridge: Cambridge University Press, 1959.

6. Fernando DMS. The detection of antisperm antibodies in the male partners of infertile couples: a protocol of sperm preparation for enhancing sperm quality. A thesis for the degree of Doctor of Philosophy, University of Colombo 2001.

Sri Lanka Journal of Obstetrics and Gynaecology 
7. Collins JA, Burrows EA, et al. Frequency and predictive value of antisperm antibodies among infertile couples. Human Reproduction 1993; 17: 592-8.

8. Esteves SC, Schneider DT, Versa Jr. S. Influence of antisperm antibodies in the semen on intracytoplasmic sperm injection outcome. International Braz J Urol 2007; 33: $795-802$.

9. Flickinger CJ, Howards SS, Barran ML, et al. Appearance of natural antisperm autoantibodies after sexual maturation of normal Lewis rats. J Reprod Immunol 1997; 33: 127-45.

10. Al-Daghistani HI, Hamad AW, Abdel-Dayem M, et al. Evaluation of serum testosterone, progesterone, seminal antisperm antibodies and fructose levels among Jordanian males with a history of infertility. Biochem Res Int 2010; Epub 409640.

11. Kalaydjiev SK, Dimitrova DK, Trifonova NL, et al. The agerelated changes in the incidence of natural antisperm antibodies suggest they are not auto/isoantibodies. Am J Reprod Immunol 2002; 47: 65-71.

12. Mazumdar S, Levine AS. Antisperm antibodies: etiology, pathogenesis, diagnosis and treatment. Ferility and Sterility 1998; 70: 799-810.

13. Shamy MY, Abdel-Hamid HA, el Gazzar R, et al. Assessment of antisperm antibody in sera of mercury and lead exposed workers. Egypt Public Health Assoc 1998; 73: $11-29$

14. Hinz S, Rajs-Bahramis S, Kempkensteffen C, et al. Effect of obesity on sex hormone levels, antisperm antibodies and fertility after vasectomy reversal. Urology 2010; 76: 851-6.

15. Fu GB, Oian LX, Cui YG, Xuan HB, Zhu JG, Zhang W, et al. Antisperm antibodies induced by testicular torsion and its influence on testicular function. Zhonghua Nan Ke Xue 2006; 13: 63-9.
16. Hirsch IH, Sedor J, Callahen HJ, Staas WE. Antisperm antibodies in seminal plasma of spinal cord-injured men. Urology 1992; 39: 243-7.

17. Djaladat H, Mahrsai A, Razazade M, et al. Varicocoele and antisperm antibody: Fact or fiction? South Med J 2006; 99: 44-7.

18. Fernando DMS, Seneviratne HR, Perera AJ. Possible causative factors for rising male infertility. Proceedings of the international congress on research in reproductive health, Mumbai 1998; abstract: 316.

19. Shiraishi $Y$, Shibahara H, Koriyama J, et al. Incidence of antisprm antibodies in males with systemic autoimmune diseases. Am J Reprod Immunol 2009; 61: 183-9.

20. Pandiyan N. Medical management of male infertility. Reproductive Biomedicine 2000; 1: 13-6.

21. Ludwikowski G,Szymanski W, Szymanski M, et al. Influence of cigarette smoking on some sperm parameters in males with decreased fertility. Przeql Lek 2004; 61: 1031-2.

22. Marinelli D, Gosparil L, Pedotti P, Taioli E. Minireview of studies on the effect of smoking and drinking habits on semen parameters. Int J Hyg Environ Health 2004; 207: 185-92.

23. Close CE, Roberts PL, Berger RE. Cigarette, alcohol and marijuana are related to pyospermia in infertile men. J Urol 1990; 144: 900-3.

24. Goldberg JM, Haering PL, Friedman CI, et al. Antisperm antibodies in women undergoing intrauterine insemination. Am J Obstet Gynecol 1990; 163: 65-8.

25. Krause WKH, Naz RK. Immune infertility - Part 2. The impact of immune reactions on human infertility. Springer Berlin Heidelberg 2009; 111-20.

26. Cunningham DS, Fulgham DL, Ray DL, et al. Antisperm antibodies to sperm surface antigens in women with genital tract infection. Am J Obstet Gynecol 1991; 164: 791-6. 